\title{
Mixing of Powders Inside a Cyclic Shear Mixing Device: A Parametric Analysis
}

\author{
Luis Guillermo Obregón ${ }^{1, *}$, Guillermo Eliecer Valencia ${ }^{2}$ and Jorge Duarte Forero ${ }^{2}$
}

\author{
${ }^{1}$ Research Group on Sustainable Chemical and Biochemical Processes, Chemical Engineering Program, Universidad del Atlántico, \\ Barranquilla, Colombia \\ ${ }^{2}$ Efficient Energy Management Research Group, Mechanical Engineering Program, Universidad del Atlántico, Barranquilla, Colombia
}

Received 3 March 2018; Accepted 12 July 2019

\begin{abstract}
Although the mixture of powders in recent years has been studied with great progress, there are still a lot of parameters to consider that make it difficult to have a complete understanding. In this work, powder mixing was studied inside a simple shear mixing device consisting of a small container with two moving walls and three static walls. The aim is to obtain a basic knowledge of the internal behavior of granular materials in the surface and the height direction of the device as a function of the particle size, filling level and disturbance of the wall velocity. Results reveal that on the surface of the cavity when velocities of the moving wall are about $9 \mathrm{~cm} / \mathrm{s}$ two zones are created, a high-speed zone and a low-speed zone. The width of the high-speed zone of flow is approximately 9 to 11 times the average particle size. A difference in density of 0.1 $\mathrm{g} / \mathrm{cm} 3$ has no significant effect on the movement of the particles from the bottom to the top. Reasons point out to the high shear stress applied by the moving walls.
\end{abstract}

Keywords: Powder Mixing, Granular Material, Filling Height, Particle Size, Shear Stress

\section{Introduction}

The powder handling industry has an incredible number of products from many areas such as chemical, pharmaceutical, metallurgical, cement, etc., [1-10]. It is necessary to know the entire process of production, the equipment used, and the operating conditions of the systems to reach the wanted quality of each granular product at the higher rate of production.

One of the most critical stages of production is the mixing process, where the homogeneity of the product is essential. There is a high number of blenders of different types used in the industry, but the most used are the tumbling blenders [11]. Valuable results have been obtained that are highly helpful when deciding on the design of this kind of mixers. However, most decisions consider only the trial and error results $[1,11$ 14]. Though lots of research have been done about tumbling blenders, such as in tote blenders [11, 15], v-blenders [16-19], double cone blenders [12, 20-22], etc., more study is needed about flow, mixing and segregation. This need points out to the different complicated flow patterns that appear when movement is applied by rotation to the blenders making difficult to have a complete and detailed study of the internal particle behavior to have a deep fundamental understanding of all the phenomena involved.

The flow pattern inside a specific system is affected by a significant number of factors such as the operating conditions [20, 23-25], e.g., rotational velocity, filling level, etc., or particle properties [26-31], e.g., particle size, repose angle, internal friction angle, cohesivity, etc., [32-34]. The absence

*E-mail address: luisobregon@mail.uniatlantico.edu.co ISSN: 1791-2377 @ 2019 School of Science, IHU. All rights reserved. doi:10.25103/iestr.124.01 of designing easy equipment that produces 3D experiments that give complete results considering all the mentioned factors prevent the acquisition of adequate data that provide a basic conception of solid mixing behavior when compared with liquid mixing behavior.

This study consisted of running a simple novel mixer that consists of an open container with two high friction moving walls with and three static ones that include the floor. The particle distribution in the surface and the depth of the cavity are studied as a function of the moving wall velocities, particle size distribution, the amplitude of moving wall, and time of mixing following a cyclic function. The aim was to have a better understanding of the physical phenomena (particle distribution, dilation, consolidation) involved in the 3-dimensional granular flow, using real granular material with polydispersity on size. The system permits targeting specific powder mixing phenomenon. Thus, better perceptions of it can be drawn from the experimental results.

\section{Experimental work}

A novel mixing device that consisted of an open box with two high friction moving walls and three static ones, as shown in Fig. 1, was used at different operating conditions to mix two granular materials with similar properties, except the color. The wall velocities were manipulated and controlled by twostep servo motors connected to a laptop in a master-slave configuration. The particles with the two different colors were loaded into the cavity and mixed under a specific shear rate. Then, the distribution of the particles was tracked as a function of the time and space using image analysis to study the mixing process. 


\subsection{Mixer}

A schematic view of the equipment which was built completely out of acrylic glass is shown in Fig. 1. The space $(W)$ between the moving walls was fixed at $2.79 \mathrm{~cm}$. The length $(L)$ and height $(H)$ of the chamber were 22.86 and $11.43 \mathrm{~cm}$, respectively. The surface of the moving walls was changed from soft to rough to produce high shear stress when having contact with the particles. It helps to create the movement to the first layer of particles, and the transfer of momentum continues being transferred to the particles in the Y-direction. The floor and the static walls in the chamber were maintained smooth.

The length of the moving walls, which was $71.12 \mathrm{~cm}$, was large enough compared to the length of the compartment $(22.86 \mathrm{~cm})$ to run experiments at different amplitudes of the periodic function used. Two DC servo motors (model Baldor BSM80C-175AA) with two shafts were used to connect the two moving walls. The software Workbench allowed to work with different speeds and amplitudes of the moving walls.

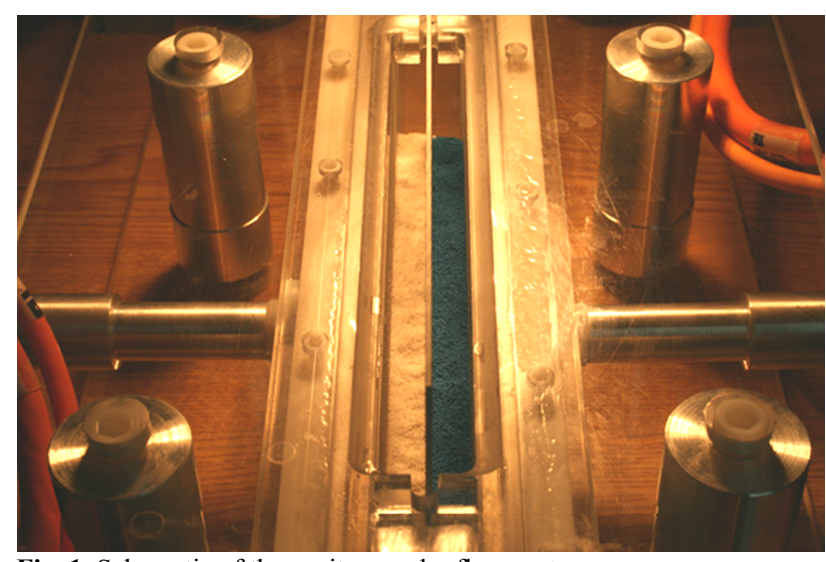

Fig. 1. Schematic of the cavity powder flow system

The cyclic function of the velocity of the moving walls is shown below in Eq. 1.

$v=\left\{\begin{array}{c}a, c m / s \rightarrow \text { if } 0<t, \mathrm{sec}<T / 2 \\ -a, c m / s \rightarrow \text { if } T / 2<t, \mathrm{sec}<T\end{array}\right.$

Where $T$ is the fundamental period.

It was used discontinuous flow, see Fig. 2. The values of the working wall velocities were established to guarantee enough transfer of momentum to produce motion to most of the particles inside the chamber.

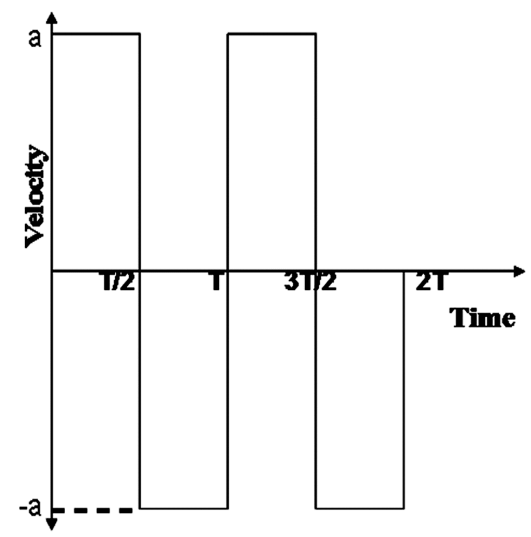

Fig. 2. Wall motions corresponding to the periodic flow

\subsection{Experimental conditions}

The operating conditions used in these experiments were, amplitude of the periodic function $\mathrm{A}=[2.24 ; 34.29 \mathrm{~cm}]$, wall velocity $\mathrm{v}=[9 ; 13 \mathrm{~cm} / \mathrm{s}]$, filling height $\mathrm{Hf}=[4.44 ; 7.62 \mathrm{~cm}]$. The speed of the walls was confirmed with a tachometer. The error of displacement measured was about $\pm 1 \mathrm{~mm}$. The moving walls always moved in the opposite direction. The powders were not hygroscopic, and their humidity was always constant to about $0.5 \% \mathrm{w} / \mathrm{w}$.

The Particle Size Distributions used are shown in Tab. 1. It includes the statistics of the particle size of both powders (dark and white).

Table 1. Particle size distribution for dark and white particles in each range of study

\begin{tabular}{c|c|c|c}
\hline \multicolumn{4}{c}{ Particle size distribution for dark particles } \\
\hline $\begin{array}{c}\text { Descriptive } \\
\text { Statistics }\end{array}$ & $\mathbf{( 3 5 5 - 4 2 5} \boldsymbol{\mu m})$ & $\mathbf{( 5 0 0 - 6 0 0 \mu m )}$ & $(\mathbf{6 0 0 - 7 1 0 \mu m )}$ \\
\hline Mean, $\mu \mathrm{m}$ & 393,6 & 575.4 & 664.45 \\
Median, $\mu \mathrm{m}$ & 391.1 & 570.5 & 663.98 \\
Standard & 52.37 & 63.4 & 67.23 \\
Deviation & 0.69 & 1.2 & 0.60 \\
Kurtosis & -0.21 & 0.4 & -0.20 \\
Skewness &
\end{tabular}

\begin{tabular}{c|c|c|c}
\hline \multicolumn{3}{c}{ Particle size distribution for white particles } \\
\hline $\begin{array}{c}\text { Descriptive } \\
\text { Statistics }\end{array}$ & $\mathbf{( 3 5 5 - 4 2 5} \boldsymbol{\mu m})$ & $\mathbf{( 5 0 0 - 6 0 0 \mu \mathbf { m } )}$ & $\mathbf{( 6 0 0 - 7 1 0} \boldsymbol{\mu m})$ \\
\hline Mean, $\mu \mathrm{m}$ & 381.26 & 545.33 & 655.73 \\
Median, $\mu \mathrm{m}$ & 379.98 & 540.39 & 657.44 \\
Standard & 71.62 & 85.17 & 87.78 \\
Deviation & 0.26 & 1.2 & 0.45 \\
Kurtosis & -0.22 & 0.3 & -0.63 \\
Skewness & &
\end{tabular}

Fig. 3 shows a sample of the shape of the white particles in the range of size from $600 \mu \mathrm{m}$ to $710 \mu \mathrm{m}$. The surface of the dark particles and the white particles of each size range of size were similar. These particle shapes were neither circular nor flat as seen in Fig.3. However, the surface has a lot of roughness that supplies enough friction to produce an excellent transfer of momentum among particles. These powders have no cohesivity, and the angle of repose of was approximately $25^{\circ}$.

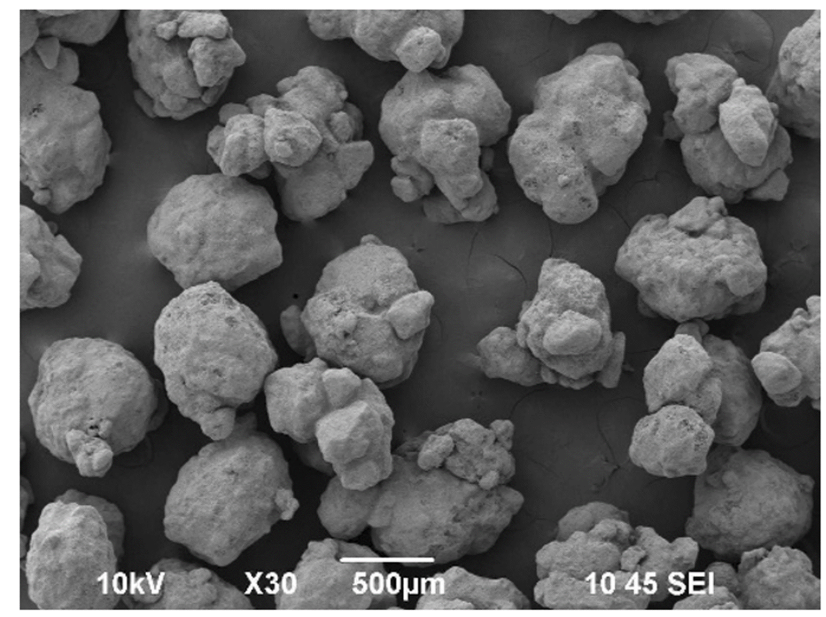

Fig. 3. White particles shape $(600-710 \mu m)$ 


\subsection{Mixing visualization}

To start the experiments, the cavity was divided into two parts by placing a divisor just in the center, parallel to the moving walls and with the same length of the cavity, $22.86 \mathrm{~cm}$. One side was poured with granulated anhydrous lactose modified with dark ink, and the other side was poured with unmodified granulated anhydrous lactose. After that, the divisor was withdrawn from the chamber, obtaining the powder arrangement seen in Fig. 1 parallel to the moving walls. When the experiments finish, a solidifier was poured slowly on the surface of the mixed powder until it infiltrates entirely to the bottom. The sample was dried for $10 \mathrm{~min}$, removed from the mixer, and placed in a hood for an entire day until being completely dried. Finally, the whole block was cut vertically in 8 slices, as shown in Fig. 4, each slice with the same length. Images of each slice were taken to analyze the particle distribution in the vertical layers ( $\mathrm{Y}-\mathrm{Z}$ planes).

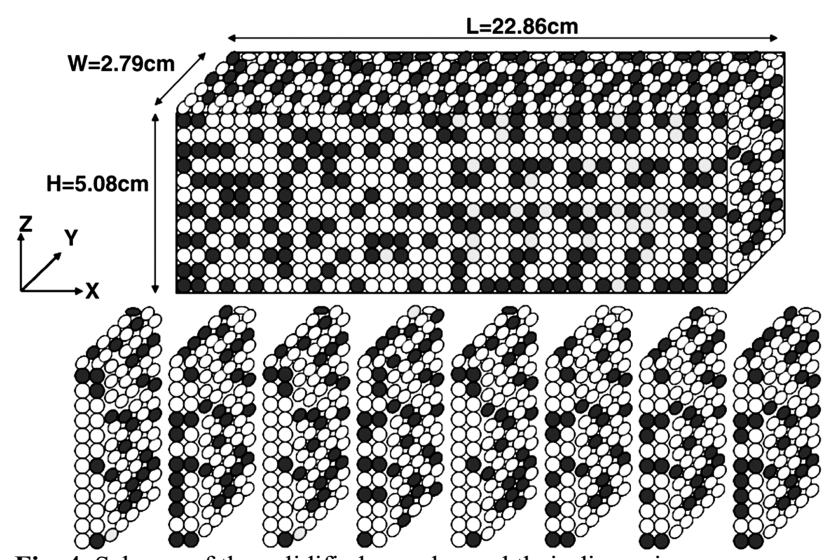

Fig. 4. Scheme of the solidified samples and their dimensions

\section{Results and Discussion}

\subsection{Filling height effect}

Figure 5 demonstrates the effect of the filling height on the mixing profile produced in the depth of the cavity (plane $y-z$ ) only at the center, at $x=L / 2$. It shows the distribution of the particles from the moving walls $(y=0$ and $y=W)$ toward the center $(y=W / 2)$ as shear was applied. The higher the filling height, the lower the triangle giving, as a result, an increase in the mixing. It suggests that the weight of the particles induce an increase in the transfer of momentum in the Z-axis.

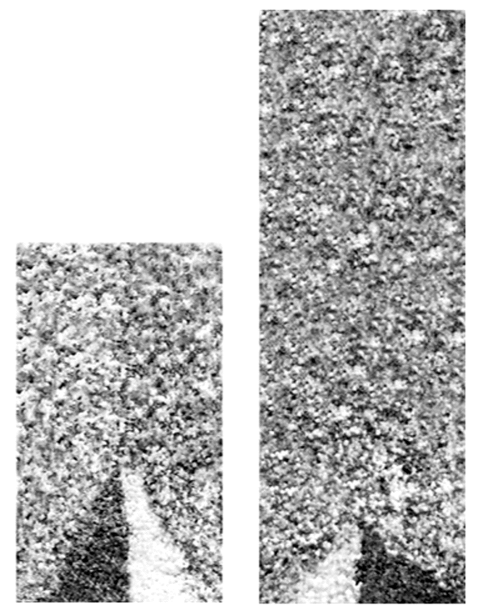

Fig. 5. Distribution of particles in the vertical layer at different filling heights, 4.44, and $7.62 \mathrm{~cm}$ from left to right. $v / W=4.65 / \mathrm{s}, t=60 \mathrm{~s}$, particle size $(600-710 \mu \mathrm{m})$, Amplitude of wall $=34.29 \mathrm{~cm}$.
The effect of the filling height can be seen as well in the surface of the cavity, as shown in Fig. 6 . The beginning shows the division of the particle exactly in the middle $(50 \%$ each side). Fig. 6 shows that the mixing increases for both experiments at different heights when the time increases. However, the homogeneity of the mixing is achieved first at a higher height.

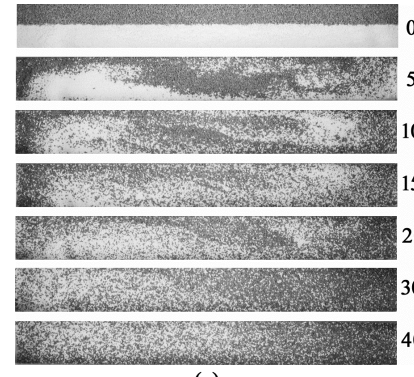

(a)

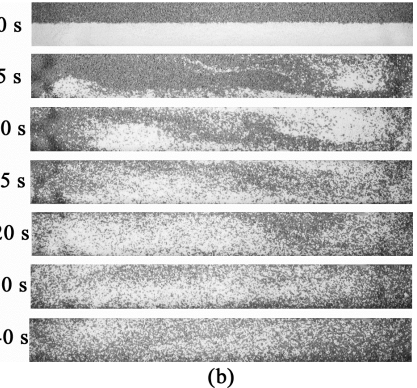

(b)
Fig. 6. Distribution of particles in the surface at different filling heights, (a) 4.44 , and (b) $7.62 \mathrm{~cm}$ from left to right. $v / W=4.65 /$ s, particle size $(600$ $710 \mu \mathrm{m})$, Amplitude of wall $=34.29 \mathrm{~cm}$.

\subsection{Particle size in the surface}

Fig. 7 shows a scheme of the picture taken to the top of the cavity (X, Y-axis) during the experiments run with two different particle sizes. When it is increased the average particle diameter from dp to Dp, as shown in Fig. 7, the zone of flow increases from $h$ to $\mathrm{H}$. All the particles in this zone corresponding to the red particles, have velocity in the $\mathrm{X}$-axis, causing a fast mixing in this zone. It can be seen that the higher the particle size, the higher the transfer of momentum to the center of the cavity. The shear stress yx(y) has a significant effect only in zones $\mathrm{H}$ and $\mathrm{h}$. In the rest of the $\mathrm{Y}$ axis, the effect is extremely slow. However, it is transmitted, causing a mixing that takes time to be homogeneous. In the zones, $\mathrm{H}$ and $\mathrm{h}$ particles move so fast that causes an increase in porosity in the $Z$-axis, causing a fast mixing because particles change their position along the $\mathrm{Z}$ and $\mathrm{Y}$-axis in those zones.

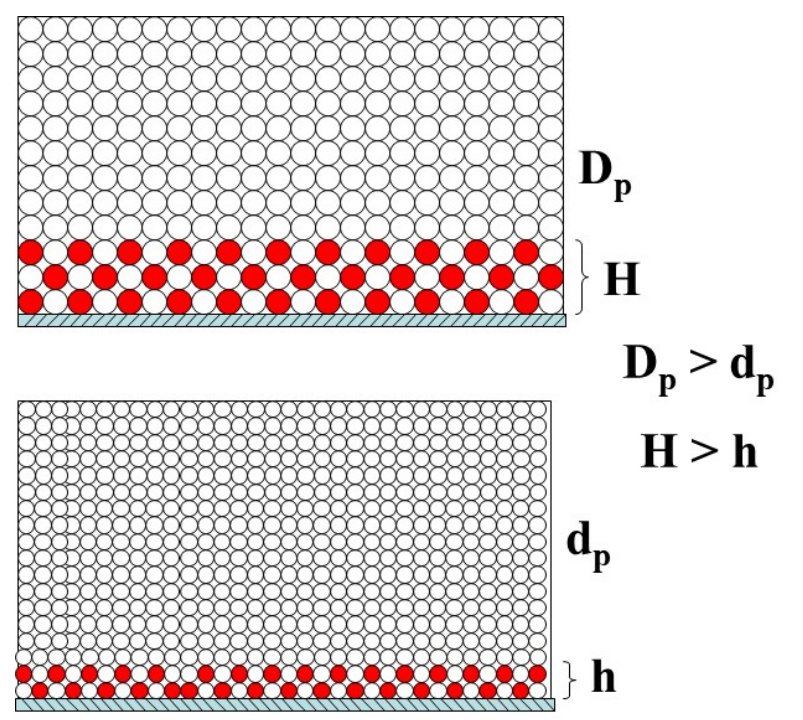

Fig. 7. Effect of particle size in the transfer of momentum on the surface of the cavity.

Fig. 8 shows the top view of the experiments made at two different range of particle sizes (500-600 m), and (355$425 \mu \mathrm{m})$. The powders were arranged perpendicular to the moving walls to see the effect of the particle size in the zone 
of mixing. Pictures of the surface were taken at a different time to observe the elongation of the division of the powders.

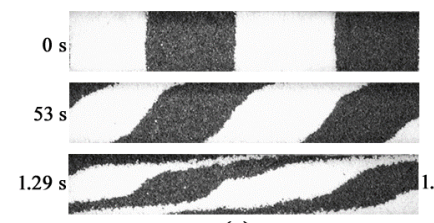

(a)

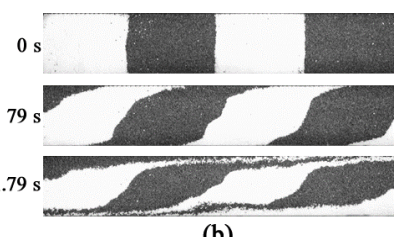

(b)
Fig. 8. Distribution of particles in the surface at different particle sizes, $(500-600 \mu \mathrm{m})$, and $(355-425 \mu \mathrm{m})$ from left to right. $v / W=3.22 / \mathrm{s}$, Amplitude of wall $=34.29 \mathrm{~cm}$.

Fig. 8a shows at time 53 seconds that the velocity profile is continuous, indicating that the transfer of momentum moves the powder as an entire solid until the static friction coefficient of the powders is overcome. However, as soon as the static friction is reached, the effect of the shear stress applied by the walls range only a specific distance. The distance that corresponds to the zone where the division of the two powders has its maximum elongation near the moving walls can be watched clearly in Fig. $8 \mathrm{a}$ at time 1.29 seconds. I another hand, Fig. $8 \mathrm{~b}$ shows at time 79 seconds that the bulk of the powders in the surface are broken in two parts. It can be happening due to the cohesivity of the powders. Small particles have higher cohesion than big particles causing a high consolidation when they are stored in a recipient. The effect of the shear stress applied on the powders in Fig. 8b reach only a small area of mixing. The particles in the center of the cavity move slow, maintaining a shape that shows the difference between Fig. 8a and 8b. The zone of slow-motion in the cavity is higher in Fig. $8 \mathrm{~b}$ than in Fig. 8a. For a given distance in the Y direction, small particles move with lower velocity reducing the transfer of momentum from the wall to the center of the cavity, reducing as well the mixing at the surface. Some experiments at different particles sizes were done, and the width corresponding to the particles having an $\mathrm{X}$-velocity was measured. Finally, it was obtained the information of Tab. 2.

Table 2. Relation between the size of the particle moving area and the particle size

\begin{tabular}{c|c}
\hline $\begin{array}{c}\text { Average particle diameter } \\
(\mathbf{D} \mathbf{p} / \boldsymbol{\mu m})\end{array}$ & $\begin{array}{c}\text { Width of the area } \\
(\mathbf{H} / \mathbf{m m})\end{array}$ \\
\hline 195 & $1-2$ \\
315 & $3-4$ \\
370 & $6-7$ \\
780 & $8-9$ \\
\hline
\end{tabular}

Summing up the information of Tab. 2 it was obtained the following expression, see Eq. 2, where $\mathrm{H}$ refers to the width of the zone of movement, and Dp is the average particle size of the powder used.

$$
H \approx(9-11) D_{P} \rightarrow v \approx 9 \mathrm{~cm} / \mathrm{s}
$$

In the width area mentioned before, the particles have an $\mathrm{X}$-velocity almost similar to the wall velocity due to the transfer of momentum. As shown in equation 1, the expression is only valid at a velocity of $9 \mathrm{~cm} / \mathrm{s}$. Experiments made at lower velocities show a negligible effect on the Yaxis, indicating that the given shear stress is so small that only the first layer of particles in the Z-X plane moves. Otherwise, when the velocity is higher than $9 \mathrm{~cm} / \mathrm{s}$ the transfer of momentum is so high that particles near the center of the cavity move with $\mathrm{X}$-velocity.

\subsection{Particle behavior in the $\mathrm{Z}$-axis}

The $X$ velocity of the walls causes not only a transfer of momentum in the $\mathrm{Y}$-axis but also in the Z-axis. This behavior causes an increase in porosity in the bulk of the powder letting the particles to be mixed in the $\mathrm{Z}$-axis as well. This phenomenon can be seen in the experiment of Fig. 9, where an experiment was done with two beds of powders, one in the bottom (dark color) and the other in the top (white color). The movement was given in the $\mathrm{x}$-axis by moving walls, and it is expected the particle to move only in that direction. However, due to the effect of the new porosity acquired in the bulk of the powders during the applied shear stress, particles of the bottom move to the top, as seen in Fig. 9.

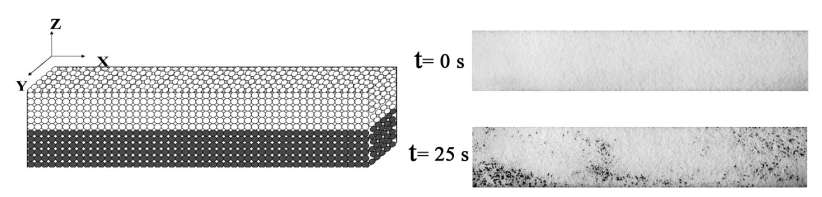

Fig.9. Distribution of particles in the Z-axis. $D p=(500-600 \mu m)$. $v / W=3.22 / \mathrm{s}$, Amplitude of wall=34.29 cm. White powder (Top), Dark powder (Bottom)

It means that the velocity profile obtained in Fig. 8 is affected by the phenomena of Fig. 9. Researchers may think that this effect is due to the difference in density between the dark particles $\left(0.6 \mathrm{~g} / \mathrm{cm}^{3}\right)$ and the white particles $\left(0.7 \mathrm{~g} / \mathrm{cm}^{3}\right)$. This parameter has a significant effect in powder mixing; the higher the difference in density, the higher its effect in the mixing, causing probable segregation. However, it was made one more experiment with the same powders arranged in contrary sides. Now the dark powder was in the top and the white in the bottom, as seen in Fig. 10.

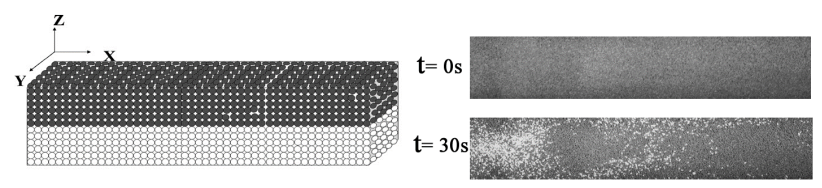

Fig. 10. Distribution of particles in the Z-axis. $D p=(500-600 \mu \mathrm{m})$. $v / W=3.22 / \mathrm{s}$, Amplitude of wall=34.29 cm. White powder (Bottom), Dark powder (Top)

Although white particles have a higher density than dark particles, many white particles move to the surface at a time of 30 seconds. The behavior obtained in Fig. 10 indicates that the effect of density is negligible compared with the transfer of momentum given to the particles by the wall.

\subsection{Effect of vibration in one of the walls}

Vibration in powders is of extreme importance because it has a high effect in the powder porosity. When vibration is imparted to one of the walls, it gets transmitted to the particles obtaining different behavior in the two mixing zones. In this case, the transfer of momentum given by the walls is higher in one of them than in the other. This difference in transfer of momentum causes the powder distribution seen in Fig. 11.

Fig. 11 shows a parabolic pattern in the bottom of the cavity. The dark powders which are in contact with the wall with no vibration lie on the withe powder. It happens because the white powder gets an extra force due to the vibration producing a higher porosity in the white zone, causing the dark powders to replace the space created by the white particles. For this reason, it shows up the parabolic behavior 


\section{Luis Guillermo Obregón, Guillermo Eliecer Valencia and Jorge Duarte Forero/}

Journal of Engineering Science and Technology Review 12 (4) (2019) 1 - 6

in the static region. With the time the parabolic shape tends to diminish as seen at time 140 seconds, and the powder above the static zone gets completely mixed.
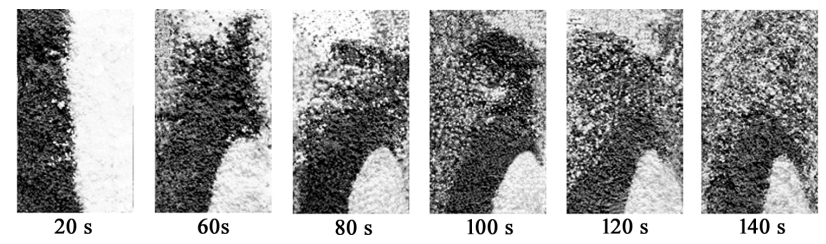

Fig. 11. Distribution of particles in the vertical layer in the middle of the cavity. $D p=(425-600 \mu \mathrm{m}) . v / W=3.22 / \mathrm{s}$, Amplitude of wall $=2.24 \mathrm{~cm}$. Vibration in the right wall.

\section{Conclusions}

A geometric shape is formed in the bottom of the cavity, $\mathrm{Y}-\mathrm{Z}$ plane, as the result of the velocity of the moving walls. This shape is triangular when both walls have the same velocity, and parabolic when one of them has a disturbance like a vibration. The size of the shape depends on parameters like the particle size, initial filling height, and velocity of the walls.

On the surface of the cavity, X-Y plane, for velocities of the moving wall about $9 \mathrm{~cm} / \mathrm{s}$ two zones are created, a highspeed zone and a low-speed zone. An increase in the particle diameter causes an increase in the width of the high-speed zone of flow approximately 9 to 11 times the average particle size.

As shear was applied by the moving walls, the effect of increasing the initial powder filling level was significant, decreasing the height of the static zone resulting in an increase in the mixing due to the increase in the transfer of momentum in the Z-axis.

The $\mathrm{X}$ velocity of the walls causes not only a transfer of momentum in the $\mathrm{Y}$-axis but also in the $\mathrm{Z}$-axis resulting in an increase in porosity in the bulk of the powder letting the particles to be mixed in the Z-axis as well.

A difference in density of $0.1 \mathrm{~g} / \mathrm{cm}^{3}$ has no significant effect on the movement of the particles from the bottom to the top. The only parameter that causes this motion in the Z-axis is the shear stress applied.

\section{Acknowledgment}

The authors appreciate the collaboration given by Professor Carlos Velazquez Figueroa from the University of Puerto Rico, Mayagüez Campus, for his advice given to achieve the best results of the project.

This is an Open Access article distributed under the terms of the Creative Commons Attribution License

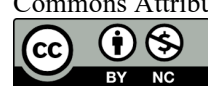

\section{References}

[1] J. Baxter, H. Abou-Chakra, U. Tuzun, and B. M. Lamptey. A DEM simulation and experimental strategy for solving fine powder flow problems. Chemical Engineering Research \& Design, 78, 1019$1025,2000$.

[2] J. Khanam and A. Nanda. Flow of granules through cylindrical hopper. Powder Technology, 150, 30-35, 2005.

[3] N. Laitinen. Opening New Perspectives for Visual Characterisation of Pharmaceutical Solids. Doctoral degree, Department of Pharmacy, University of Helsinki, Helsinki, 2003.

[4] B. Laurent. Characterisation of wet-mass granulation. european pharmaceutical review, 95-99, 2004.

[5] H. Li and J. J. McCarthy, Cohesive particle mixing and segregation under shear. Powder Technology, 164, 58-64, 2006.

[6] L. Obregon and C. Velazquez. Discrimination limit between mean gray values for the prediction of powder concentrations. Powder Technology, 175, 8-13, 2007.

[7] A. Realpe and C. Velazquez. Image processing and analysis for determination of concentrations of powder mixtures. Powder Technology, 134, 193-200, 2003.

[8] M. J. P. Hiseman, B. F. C. Laurent, J. Bridgwater, D. I. Wilson, D. J. Parker, N. North, et al. Granular flow in a planetary mixer. Chemical Engineering Research \& Design, 80, 432-440, 2002.

[9] G. Metcalfe and M. Shattuck. Pattern formation during mixing and segregation of flowing granular materials. Physica A, 233, 709-717, 1996.

[10]N. Jain, J. M. Ottino, and R. M. Lueptow. Effect of interstitial fluid on a granular flowing layer. Journal of Fluid Mechanics, 508, 23-44, 2004.

[11]P. E. Arratia, N.-h. Duong, F. J. Muzzio, P. Godbole, and S. Reynolds. A study of the mixing and segregation mechanisms in the Bohle Tote blender via DEM simulations. Powder Technology, 164, 50-57, 2006.

[12] M. Moakher, T. Shinbrot, and F. J. Muzzio. Experimentally validated computations of flow, mixing and segregation of non-cohesive grains in 3D tumbling blenders. Powder Technology, 109, 58-71, 2000.

[13]R. L. Stewart, J. Bridgwater, and D. J. Parker. Granular flow over a flat-bladed stirrer. Chemical Engineering Science, 56, 4257-4271, 2001.

[14] J. Bridgwater, S. Forrest, and D. J. Parker. PEPT for agglomeration?. Powder Technology, 140, 187-193, 2004.
[15]O. S. Sudah, D. Coffin-Beach, and F. J. Muzzio. Quantitative characterization of mixing of free-flowing granular material in tote (bin)-blenders. Powder Technology, 126, 191-200, 2002.

[16] A. Alexander, F. J. Muzzio, and T. Shinbrot. Segregation patterns in V-blenders. Chemical Engineering Science, 58, 487-496, 2003.

[17]J. Doucet, F. Bertrand, and J. Chaouki. Experimental characterization of the chaotic dynamics of cohesionless particles: Application to a V-blender. Granular Matter, 10, 133-138, 2008.

[18] M. Lemieux, G. Leonard, J. Doucet, L. A. Leclaire, F. Viens, J. Chaouki, et al. Large-scale numerical investigation of solids mixing in a V-blender using the discrete element method. Powder Technology, 181, 205-216, 2008.

[19]H. P. Kuo, P. C. Knight, D. J. Parker, Y. Tsuji, M. J. Adams, and J. P. K. Seville, The influence of DEM simulation parameters on the particle behaviour in a V-mixer. Chemical Engineering Science, 57, 3621-3638, 2002.

[20]D. Brone and F. J. Muzzio. Enhanced mixing in double-cone blenders. Powder Technology, 110, 179-189, 2000.

[21]A. W. Chester, J. A. Kowalski, M. E. Coles, E. L. Muegge, F. J. Muzzio, and D. Brone. Mixing dynamics in catalyst impregnation in double-cone blenders. Powder Technology, 102, 85-94, 1999.

[22]A. W. Alexander, T. Shinbrot, and F. J. Muzzio. Granular segregation in the double-cone blender: Transitions and mechanisms. Physics of Fluids, 13, 578-587, 2001.

[23]Y. C. Zhou, A. B. Yu, R. L. Stewart, and J. Bridgwater. Microdynamic analysis of the particle flow in a cylindrical bladed mixer. Chemical Engineering Science, 59, 1343-1364, 2004.

[24]G. Hu, X. Gong, H. Huang, and Y. Li. Effects of geometrie parameters and operating conditions on granular flow in a modified rotating cone. Industrial and Engineering Chemistry Research, 46, 9263-9268, 2007.

[25] J. Olinek, C. Anand, and C. T. Bellehumeur. Experimental study on the mixing and segregation of granular particles in rotational molding. Chicago, IL., United States, 2004, 860-864.

[26]B. Chaudhuri, A. Mehrotra, F. J. Muzzio, and M. S. Tomassone. Cohesive effects in powder mixing in a tumbling blender. Powder Technology, 165, 105-114, 2006.

[27] S.-S. Hsiau, J.-Y. Shiu, W.-L. Yang, and L.-S. Lu. Influence of internal friction on transport properties in sheared granular flows. Aiche Journal, 52, 3592-3599, 2006. 


\section{Luis Guillermo Obregón, Guillermo Eliecer Valencia and Jorge Duarte Forero/}

Journal of Engineering Science and Technology Review 12 (4) (2019) 1 - 6

[28]C. K. K. Lun, Kinetic theory for granular flow of dense, slightly inelastic, slightly rough spheres. Journal of Fluid Mechanics, 233, 539-559, 1991.

[29] M. K. Mazumder, J. R. Mountain, T. Chasser, and P. Pettit, Fluidity and flow properties of fine powders. Particulate Science and Technology, 15, 106, 1997.

[30]D. Z. Zhang and R. M. Rauenzahn. Effects of long and short relaxation times of particle interactions in dense and slow granular flows. Honolulu, HI, United States, 2003, 579-582.

[31] Y. C. Zhou, B. H. Xu, A. B. Yu, and P. Zulli. An experimental and numerical study of the angle of repose of coarse spheres. Powder Technology, 125, 45-54, 2002.
[32]L. G. Obregon, M. E. Robledo, and C. Velazquez. Simulation of a Compressible Powder Flow under Oscillatory Shear Stress Modeled as a Non - Linear Fluid by Using an Explicit Solution Method. Journal of Engineering Science and Technology Review, 11, 84-90, 2018 .

[33]A. M. Fuentes, J. B. Restrepo, and L. G. Obregon. Comparative Study of Mixing Liquids and Powders in a Continuous Medium using CFD. Journal of Engineering Science and Technology Review, 11, 134-138, 2018.

[34]L. Obregon, A. Realpe, and C. Velazquez. Mixing of granular materials, part II: effect of particle size under periodic shear. Powder Technology, 201, 193-200, 2010. 